\title{
Pacific
}

Journal of

Mathematics

\section{PREPOLAR DEFORMATIONS AND A NEW LÊ-IOMDINE} FORMULA

DAVID MASSEY 


\title{
PREPOLAR DEFORMATIONS AND A NEW LÊ-IOMDINE FORMULA
}

\author{
DAVID B. MASSEY
}

\begin{abstract}
In this short paper, we wish to describe a class of deformations of affine hypersurface singularities. These deformations, which we call prepolar deformations, are the ones which we found to be important in our work on the Lê numbers of a hypersurface singularity. However, the results of this paper are not directly related to our work on the Lê numbers.
\end{abstract}

\section{Introduction.}

The main result of this paper is to use prepolar deformations to put lower bounds on the top Betti number of the Milnor fibre of a non-isolated hypersurface singularity. These lower bounds are a corollary of a generalization of the formula of Lê and Iomdine.

Let $\mathcal{U}$ be an open neighborhood of the origin in $\mathbb{C}^{n+1}$, let $f:(\mathcal{U}, \mathbf{0}) \rightarrow$ $(\mathbb{C}, 0)$ be an analytic function, and let $\Sigma f$ denote the critical locus of $f$. Let $L: \mathbb{C}^{n+1} \rightarrow \mathbb{C}$ be a generic linear form (we need for $L$ to be prepolar with respect to $f$; we shall define this in section 2 ).

The formula of Lê and Iomdine says that, if $\operatorname{dim}_{0} \Sigma f=1$, then, for all large $j, f+L^{j}$ has an isolated singularity at the origin and

$$
b_{n}\left(f+L^{j}\right)=\mu\left(f+L^{j}\right)=b_{n}(f)-b_{n-1}(f)+j \sum_{\nu} m_{\nu} \delta_{\nu}(f),
$$

where $b_{\imath}()$ denotes the $i$-th Betti number of the Milnor fibre of a function at the origin, $\mu$ denotes the Milnor number of the isolated singularity at the origin, the summation is over all components, $\nu$, of $\Sigma f, m_{\nu}$ is the local degree of $L$ restricted to $\nu$ at the origin, and $\delta_{\nu}(f)$ is the Milnor number of a generic hyperplane slice of $f$ at a point $\mathbf{p} \in \nu-\mathbf{0}$ sufficiently close to the origin.

This formula has, at least, two possible generalizations. One generalization is in terms of Lê numbers; this is the formula which appears in [Mas1], [Mas3], and [M-S]. But, while there are Morse inequalities between the Lê numbers and the Betti numbers of the Milnor fibre, the Lê numbers are not themselves (generally) Betti numbers of the Milnor fibre. So, one might ask 
for a generalization of the formula of Lê and Iomdine which generalizes the Betti number information.

In this paper, we prove that, if $\operatorname{dim}_{0} \Sigma f=s \geqslant 1$, then, for all large $j$, $\operatorname{dim}_{0} \Sigma\left(f+L^{j}\right)=s-1$ and

$$
b_{n}\left(f+L^{j}\right)=b_{n}(f)-b_{n-1}(f)+j\left(b_{n-1}\left(f_{\mid V(L)}\right)-\left(\Gamma_{f, L}^{1} \cdot V(L)\right)_{0}\right),
$$

where $\Gamma_{f, L}^{1}$ denotes the relative polar curve ([L-T], [Te1], [Te2]) of $f$ with respect to $L$ and (.) denotes the scheme-theoretic intersection number. In the case where $f$ has a one-dimensional critical locus at the origin, it is easy to show that this new formula reduces to that of Lê and Iomdine.

We consider this new Lê-Iomdine formula interesting because it implies that

$$
b_{n-1}\left(f_{\left.\right|_{V(L)}}\right) \geqslant\left(\Gamma_{f, L}^{1} \cdot V(L)\right)_{0} .
$$

In terms of deformations, this says that the top possible non-zero Betti number of the Milnor fibre of $f_{\mid v(L)}$ is greater than or equal to $\left(\Gamma_{f, L}^{1} \cdot V(L)\right)_{0}$ for any $f$ which has $V(L)$ as a prepolar slice. Thus, if we define $f$ to be a prepolar deformation of $f_{\mid V(L)}$ precisely when $V(L)$ is a prepolar slice of $f$, we obtain a class of deformations of $f_{\left.\right|_{V(L)}}$ which give lower bounds on the top Betti number of the Milnor fibre. This also suggests that it might be useful to study prepolar deformations for which $\left(\Gamma_{f, L}^{1} \cdot V(L)\right)_{0}$ obtains its maximum value.

\section{The Relative Polar Curve.}

Throughout this section, $\mathcal{U}$ will denote an open subset of $\mathbb{C}^{n+1}$ containing the origin. We denote the coordinates of $\mathbb{C}^{n+1}$ by $\left(z_{0}, \ldots, z_{n}\right)$. We use vertical lines, $\|$, to denote the underlying set of an analytic scheme.

Let $W$ be analytic subset of $\mathcal{U}$ and let $\alpha$ be a coherent sheaf of ideals in $\mathcal{O}_{u}$. At each point, $\mathbf{x}$, of $V(\alpha)$, we wish to consider scheme-theoretically those components of $V(\alpha)$ which are not contained in the set $|W|$.

Definition 1.1. Let $A$ denote $\mathcal{O}_{u, \mathbf{x}}$; we write $\alpha_{\mathbf{x}}$ for the stalk of $\alpha$ in $A$. Let $\mathrm{S}$ be the multiplicatively closed set $A-\bigcup p$ where the union is over all prime ideals $p \in A s s\left(A / \alpha_{\mathbf{x}}\right)$ with $|V(p)| \nsubseteq|W|$. Then, we define $\alpha_{\mathbf{x}} / W$ to equal $S^{-1} \alpha_{\mathbf{x}} \cap A$.

Thus, $\alpha_{\mathbf{x}} / W$ is the ideal in $A$ consisting of the intersection of those primary components, $q$, (possibly embedded), of $\alpha_{\mathbf{x}}$ such that $|V(q)| \nsubseteq|W|$.

Now, we have defined $\alpha_{\mathbf{x}} / W$ in each stalk. By [Si-Tr], if we perform this operation simultaneously at all points of $V(\alpha)$, then we obtain a coherent sheaf of ideals called a gap sheaf; we write this sheaf as $\alpha / W$. If $V=V(\alpha)$, we let $V / W$ denote the scheme $V(\alpha / W)$. 
It is important to note that the scheme $V / W$ does not depend on the structure of $W$ as a scheme, but only as an analytic set.

The following lemma is very useful for calculating $V / W$, and is merely an exercise in localization (see [Mas2] and [Mas3]).

Lemma 1.2. Let $\left(X, \mathcal{O}_{x}\right)$ be an analytic space, let $\alpha, \beta$, and $\gamma$ be coherent sheaves of ideals in $\mathcal{O}_{x}$, let $f, g \in \mathcal{O}_{x}$, and let $W$ be an analytic subset of $X$.

If $V(\alpha+\gamma) \subseteq W$, then $((\alpha \cap \beta)+\gamma) / W=(\beta+\gamma) / W$, and thus

$$
((V(\alpha) \cup V(\beta)) \cap V(\gamma)) / W=(V(\beta) \cap V(\gamma)) / W
$$

as schemes.

We will now use these gap sheaves to define the relative polar curve of a map [L-T2], [Te2], [Te3] with respect to a linear form. Let

$$
f:(\mathcal{U}, \mathbf{0}) \rightarrow(\mathbb{C}, \mathbf{0})
$$

be an analytic map, let $\Sigma f$ denote its critical locus, and let $L: \mathbb{C}^{n+1} \rightarrow \mathbb{C}$ be a linear form.

Definition 1.3. After a linear change of coordinates, we may use $\left(L, z_{1}, \ldots, z_{n}\right)$ as coordinates on $\mathcal{U}$. Using these coordinates, we define the relative polar curve of $f$ with respect to $L$ to be the subscheme of $\mathcal{O}_{\mathcal{U}}$ defined by

$$
\Gamma_{f, L}^{1}:=V\left(\frac{\partial f}{\partial z_{1}}, \ldots, \frac{\partial f}{\partial z_{n}}\right) / \Sigma f
$$

(again, see [Mas1], [Mas2], [Mas3]). Thus, on the level of defining ideals, $\Gamma_{f, L}^{1}$ consists of those components of $V\left(\frac{\partial f}{\partial z_{1}}, \ldots, \frac{\partial f}{\partial z_{n}}\right)$ which are not contained in $|\Sigma f|$. The key point of this definition is that the dimension of the singular set of $f$ is allowed to be arbitrary.

Of course, for arbitrary $L, \Gamma_{f, L}^{1}$ need not actually be a curve, even as a germ at the origin. However, as we shall see in the next section, if $L$ is prepolar with respect to $f$ at the origin - a genericly satisfied condition - then $\Gamma_{f, L}^{1}$ is, in fact, one-dimensional (or empty) at the origin. Moreover, it follows immediately from the definition that whenever $\Gamma_{f, L}^{1}$ is one-dimensional at the origin, $\Gamma_{f, L}^{1} \cap V\left(\frac{\partial f}{\partial L}\right)$ is zero-dimensional or empty at the origin.

When we take intersection numbers of schemes in this paper, we are of course actually taking the intersection numbers of the associated cycles. Thus, it will be clear from the context what structure we are using for the polar curve, and we need not introduce any extra notation to distinguish the cycle from the scheme. 


\section{Good Stratifications and Prepolar Deformations.}

As in the last section, we continue with $\mathcal{U}$ being an open subset of $\mathbb{C}^{n+1}$ containing the origin, and $f:(\mathcal{U}, \mathbf{0}) \rightarrow(\mathbb{C}, \mathbf{0})$ being an analytic map.

In [H-L], a good stratification for $f$ at $\mathbf{0}$ is an analytic stratification, $\mathcal{S}$, of $V(f)$ in a neighborhood of the origin such that: if $\mathbf{p} \in S \in \mathcal{S}$, and we have a sequence of $\mathbf{p}_{i} \in \mathcal{U}-\Sigma f$ with $\mathbf{p}_{i} \rightarrow \mathbf{p}$ and $T_{\mathbf{p}_{i}} V\left(f-f\left(\mathbf{p}_{i}\right)\right) \rightarrow \mathcal{T}$, then $T_{\mathbf{p}} S \subseteq \mathcal{T}$. We always assume that the smooth part of $V(f)$ has been chosen as a good stratum. Hamm and Lê prove in $[\mathbf{H}-\mathbf{L}]$ that good stratifications always exist.

Definition 2.1. Let $\mathcal{S}$ be a good stratification for $f$ at $\mathbf{0}$. A prepolar slice of $f$ at $\mathbf{0}$ with respect to $\mathcal{S}$ is a hyperplane $H$ such that $H$ transversely intersects each stratum of $\mathcal{S}$ in a neighborhood of the origin except, perhaps, at the origin itself.

We say simply that $H$ is a prepolar slice of $f$ at $\mathbf{0}$ if there exists a good stratification for $f$ at the origin with respect to which $H$ is prepolar. For a fixed $f$, prepolar slices are generic (see $[\mathbf{H}-\mathbf{L}],[\mathbf{M a s} 3])$.

We define prepolar deformations in the obvious manner. If $\mathcal{W}$ is an open neighborhood of the origin in $\mathbb{C}^{n}$ and $f_{0}:(\mathcal{W}, 0) \rightarrow(\mathbb{C}, 0)$ is an analytic function, then a prepolar deformation of $f_{0}$ consists of an open neighborhood, $\mathcal{U}$, of the origin in $\mathbb{C}^{n+1}$ such that $\left(0 \times \mathbb{C}^{n}\right) \cap \mathcal{U}=0 \times \mathcal{W}$ and an analytic function $f:(\mathcal{U}, \mathbf{0}) \rightarrow(\mathbb{C}, 0)$ such that $0 \times \mathbb{C}^{n}$ is a prepolar slice of $f$ at $\mathbf{0}$ and $f_{l_{0 \times \mathcal{W}}}=f_{0}$. That is, a prepolar deformation is a one-parameter deformation which has the original function as a prepolar slice.

Our primary interest in prepolar slices is due to the following result of Lê:

Theorem 2.2 [Lê1], [Mas1]. If $L: \mathbb{C}^{n+1} \rightarrow \mathbb{C}$ is a linear form such that $V(L)$ is a prepolar slice for $f$ at the origin, then $\Gamma_{f, L}^{1}$ is one-dimensional or empty at the origin, $\Gamma_{f, L}^{1} \cap V(f)$ is zero-dimensional or empty at the origin, and

$$
b_{n}(f)-b_{n-1}(f)+b_{n-1}\left(f_{\mid V(L)}\right)=\left(\Gamma_{f, L}^{1} \cdot V(f)\right)_{0},
$$

where $b_{i}()$ denotes the $i$-th Betti number of the Milnor fibre at the origin and $(\cdot)$ denotes the scheme-theoretic intersection number.

\section{A New General Lê-Iomdine Formula.}

As before, we let $\mathcal{U}$ be an open subset of $\mathbb{C}^{n+1}$ containing the origin, and let $f:(\mathcal{U}, \mathbf{0}) \rightarrow(\mathbb{C}, \mathbf{0})$ be an analytic map. Also, we use the coordinates $\left(z_{0}, \ldots, z_{n}\right)$ on $\mathcal{U}$. 
We now take a new variable, $u$, and would like to apply 2.2 to the function $f+u^{j}:(\mathbb{C} \times \mathcal{U}, \mathbf{0}) \rightarrow(\mathbb{C}, 0)$ and the hyperplane slice $V\left(z_{0}-u\right)$. This is a desirable thing to do because the Milnor fibre of $f+u^{j}$ has the homotopytype of the one-point union of $j-1$ copies of the suspension of the Milnor fibre of $f$ [Mas3], [Ok], [Sak], [Se-Th]. We first need to show that $V\left(z_{0}\right)$ being a prepolar slice for $f$ implies that $V\left(u-z_{0}\right)$ is a prepolar slice for $f+u^{j}-$ at least for all large $j$.

Proposition 3.1. If $j \geqslant 2$ and $\mathcal{S}$ is a good stratification for $f$ at the origin, then

$$
\left\{V\left(f+u^{j}\right)-\Sigma\left(f+u^{j}\right)\right\} \cup\{0 \times S \mid S \text { is a singular stratum of } \mathcal{S}\}
$$

is a good stratification for $f+u^{j}$ at the origin.

Proof. As $j \geqslant 2, \Sigma\left(f+u^{j}\right)=\{0\} \times \Sigma f$.

Let $\mathbf{p}=(0, \mathbf{q}) \in\{0\} \times \Sigma f$, where $S \in \mathcal{S}$, and let $\mathbf{p}_{i}=\left(u_{i}, \mathbf{q}_{i}\right)$ be a sequence of points in $\mathbb{C} \times \mathcal{U}-\{0\} \times \Sigma f$ such that $\mathbf{p}_{i} \rightarrow \mathbf{p}$ and $T_{\mathbf{p}_{i}} V\left(f+u^{j}-\left(f+u^{j}\right)_{\left.\right|_{\mathbf{p}_{i}}}\right) \rightarrow$ $\mathcal{T}$. We wish to show that $T_{\mathbf{p}}(\{0\} \times S)=\{0\} \times T_{\mathbf{q}} S \subseteq \mathcal{T}$.

If $\mathcal{T}=T_{\mathbf{p}} V(u)=\{0\} \times \mathbb{C}^{n+1}$, then we are finished. So, suppose otherwise. Then, by taking a subsequence, we may assume that $\mathbf{q}_{i} \notin \Sigma f$ and that $T_{\mathbf{q}_{i}} V\left(f-f\left(\mathbf{q}_{i}\right)\right) \rightarrow \eta$.

As $\mathcal{T}$ transversely intersects $T_{\mathbf{p}} V(u), T_{\mathbf{p}_{i}} V\left(f+u^{j}-\left(f+u^{j}\right)_{\left.\right|_{\mathbf{p}_{i}}}\right)$ transversely intersects $T_{\mathbf{p}_{\mathbf{i}}} V\left(u-u_{i}\right)$ for all $\mathbf{p}_{i}$ close to $\mathbf{p}$. Thus,

$$
\mathcal{T} \cap\left(\{0\} \times \mathbb{C}^{n+1}\right)=\lim T_{\mathbf{p}_{i}} V\left(f+u^{j}-\left(f+u^{j}\right)_{\left.\right|_{\mathbf{p}_{i}}}\right) \cap T_{\mathbf{p}_{i}} V\left(u-u_{i}\right)=
$$

$\lim T_{\mathbf{p}_{\boldsymbol{i}}} V\left(f+u^{j}-\left(f+u^{j}\right)_{\left.\right|_{\mathbf{p}_{i}}}, u-u_{i}\right)=\lim T_{\mathbf{p}_{i}} V\left(f-f\left(\mathbf{q}_{i}\right), u-u_{i}\right)=\{0\} \times \eta$.

Now, as $S$ is a good stratum for $f, T_{\mathbf{q}}(S) \subseteq \eta$ and the desired conclusion follows.

Corollary 3.2. If $V\left(z_{0}\right)$ is a prepolar slice for $f$ at $\mathbf{0}$ then, for all large $j$, $V\left(z_{0}-u\right)$ is a prepolar slice for $f+u^{j}$ at $\mathbf{0}$.

Proof. In light of the proposition, all that we must show is that, for all large $j, \Sigma\left(f+u^{j}\right) \cap V\left(z_{0}-u\right)=\Sigma\left(f+u_{\left.\left.\right|_{V\left(z_{0}-u\right)}\right)}\right)$.

Now,

$$
\Sigma\left(f+u^{j}\right) \cap V\left(z_{0}-u\right)=(\{0\} \times \Sigma f) \cap V\left(z_{0}-u\right)=\{0\} \times\left(\Sigma f \cap V\left(z_{0}\right)\right) .
$$

On the other hand,

$$
\Sigma\left(f+u_{\left.\right|_{V\left(z_{0}-u\right)} ^{j}}\right)=\left(\mathbb{C} \times \Sigma\left(f+z_{0}^{j}\right)\right) \cap V\left(z_{0}-u\right) .
$$


But, near the origin and for large $j, \Sigma\left(f+z_{0}^{j}\right)=\Sigma f \cap V\left(z_{0}\right)$ by Lemma 3.1 of [Mas2]. The conclusion follows.

We are now in a position to apply 2.2 to $f+u^{j}$ and the hyperplane slice $V\left(z_{0}-u\right)$. First, however, we will calculate the intersection number $\left(\Gamma_{f+u^{j}, z_{0}-u}^{1} \cdot V\left(f+u^{j}\right)\right)_{0}$.

Proposition 3.3. If $V\left(z_{0}\right)$ is a prepolar slice for $f$ at $\mathbf{0}$ then, for all large $j$,
i) $\quad \Gamma_{f+u^{j}, z_{0}-u}^{1}=\left(\mathbb{C} \times \Gamma_{f, z_{0}}^{1}\right) \cdot V\left(\frac{\partial f}{\partial z_{0}}+j u^{j-1}\right)$ as 1-cycles;
ii) $\quad\left(\Gamma_{f+u^{j}, z_{0}-u}^{1} \cdot V\left(z_{0}-u\right)\right)_{0}=\left(\Gamma_{f, z_{0}}^{1} \cdot V\left(\frac{\partial f}{\partial z_{0}}\right)\right)_{0}$;
iii) $\left(\Gamma_{f+u^{j}, z_{0}-u}^{1} \cdot V\left(\frac{\partial\left(f+u^{j}\right)}{\partial\left(z_{0}-u\right)}\right)\right)_{0}=(j-1)\left(\Gamma_{f, z_{0}}^{1} \cdot V\left(\frac{\partial f}{\partial z_{0}}\right)\right)_{0}$;
iv) $\quad\left(\Gamma_{f+u^{j}, z_{0}-u}^{1} \cdot V\left(f+u^{j}\right)\right)_{0}=j\left(\Gamma_{f, z_{0}}^{1} \cdot V\left(\frac{\partial f}{\partial z_{0}}\right)\right)_{0}$;
v) if $s:=\operatorname{dim}_{0} \Sigma f \geqslant 1$, then $\operatorname{dim}_{0} \Sigma\left(f+z_{0}^{j}\right)=s-1$.

Proof.

i) Using the coordinate system $\left(z_{0}-u, z_{0}, \ldots, z_{n}\right)$, we see that

$$
\begin{gathered}
\Gamma_{f+u^{j, z_{0}-u}}^{1}=V\left(\frac{\partial f}{\partial z_{0}}+j u^{j-1}, \frac{\partial f}{\partial z_{1}}, \ldots, \frac{\partial f}{\partial z_{n}}\right) / \Sigma\left(f+u^{j}\right)= \\
{\left[V\left(\frac{\partial f}{\partial z_{0}}+j u^{j-1}\right) \cap \mathbb{C} \times\left(\Gamma_{f, z_{0}}^{1} \cup R\right)\right] /\{0\} \times \Sigma f}
\end{gathered}
$$

where $R$ consists of the union of the primary components of $V\left(\frac{\partial f}{\partial z_{1}}, \ldots, \frac{\partial f}{\partial z_{n}}\right)$ which are contained in $\Sigma f$ (for some primary decomposition of $\left.\left\langle\frac{\partial f}{\partial z_{1}}, \ldots, \frac{\partial f}{\partial z_{n}}\right\rangle\right)$. Regardless of the decomposition, $R \subseteq \Sigma f$ and so $(\mathbb{C} \times$ $R) \cap V\left(\frac{\partial f}{\partial z_{0}}+j u^{j-1}\right) \subseteq\{0\} \times \Sigma f$. Therefore, by 1.2 ,

$$
\Gamma_{f+u^{j, z_{0}-u}}^{1}=\left[V\left(\frac{\partial f}{\partial z_{0}}+j u^{j-1}\right) \cap\left(\mathbb{C} \times \Gamma_{f, z_{0}}^{1}\right)\right] /\{0\} \times \Sigma f .
$$


But, if $C$ were a component of $V\left(\frac{\partial f}{\partial z_{0}}+j u^{j-1}\right) \cap\left(\mathbb{C} \times \Gamma_{f, z_{0}}^{1}\right)$ contained in $\{0\} \times \Sigma f$, then

$$
\begin{gathered}
C \subseteq V\left(\frac{\partial f}{\partial z_{0}}+j u^{j-1}\right) \cap\left(\mathbb{C} \times \Gamma_{f, z_{0}}^{1}\right) \cap(\{0\} \times \Sigma f)= \\
\left(\{0\} \times \Gamma_{f, z_{0}}^{1}\right) \cap\left(\{0\} \times V\left(\frac{\partial f}{\partial z_{0}}\right)\right),
\end{gathered}
$$

which is impossible as $\Gamma_{f, z_{0}}^{1} \cap V\left(\frac{\partial f}{\partial z_{0}}\right)$ is 0-dimensional.

Thus, up to embedded subvariety,

$$
\Gamma_{f+u^{j}, z_{0}-u}^{1}=V\left(\frac{\partial f}{\partial z_{0}}+j u^{j-1}\right) \cap\left(\mathbb{C} \times \Gamma_{f, z_{0}}^{1}\right)
$$

and so these schemes define the same 1-cycle (we know that $\Gamma_{f+u^{j}, z_{0}-u}^{1}$ is 1-dimensional since $V\left(z_{0}-u\right)$ is a prepolar slice of $\left.f+u^{j}\right)$. That this 1cycle equals $V\left(\frac{\partial f}{\partial z_{0}}+j u^{j-1}\right) \cdot\left(\mathbb{C} \times \Gamma_{f, z_{0}}^{1}\right)$ follows from [Fu, Prop. 7.1.b], since $\mathbb{C} \times \Gamma_{f, z_{0}}^{1}$ has no embedded subvarieties and $V\left(\frac{\partial f}{\partial z_{0}}+j u^{j-1}\right)$ contains no components of $\mathbb{C} \times \Gamma_{f, z_{0}}^{1}$. This proves $\mathrm{i}$ ).

ii) By i),

$$
\begin{aligned}
\left(\Gamma_{f+u^{j}, z_{0}-u}^{1} \cdot V\left(z_{0}-u\right)\right)_{0} & \\
= & \left(V\left(\frac{\partial f}{\partial z_{0}}+j u^{j-1}\right) \cdot\left(\mathbb{C} \times \Gamma_{f, z_{0}}^{1}\right) \cdot V\left(z_{0}-u\right)\right)_{\mathbf{0}} \\
= & \left(\left(\mathbb{C} \times V\left(\frac{\partial f}{\partial z_{0}}+j z_{0}^{j-1}\right)\right) \cdot\left(\mathbb{C} \times \Gamma_{f, z_{0}}^{1}\right) \cdot V\left(z_{0}-u\right)\right)_{\mathbf{0}} \\
= & \left(\mathbb{C} \times\left(V\left(\frac{\partial f}{\partial z_{0}}+j z_{0}^{j-1}\right) \cdot \Gamma_{f, z_{0}}^{1}\right) \cdot V\left(z_{0}-u\right)\right)_{\mathbf{0}} \\
= & \left(V\left(\frac{\partial f}{\partial z_{0}}+j z_{0}^{j-1}\right) \cdot \Gamma_{f, z_{0}}^{1}\right)_{0} .
\end{aligned}
$$

But, for all sufficiently large $j$, this last intersection number equals

$$
\left(\Gamma_{f, z_{0}}^{1} \cdot V\left(\frac{\partial f}{\partial z_{0}}\right)\right)_{0}
$$


iii) Again using i) and the coordinate system $\left(z_{0}-u, z_{0}, \ldots, z_{n}\right)$,

$$
\begin{aligned}
\left(\Gamma_{f+u^{j}, z_{0}-u}^{1} \cdot V\left(\frac{\partial\left(f+u^{j}\right)}{\partial\left(z_{0}-u\right)}\right)\right)_{\mathbf{0}} & \left(\Gamma_{f+u^{j, z_{0}-u}}^{1} \cdot V\left(j u^{j-1}\right)\right)_{\mathbf{0}} \\
= & \left(V\left(\frac{\partial f}{\partial z_{0}}+j u^{j-1}\right) \cdot\left(\mathbb{C} \times \Gamma_{f, z_{0}}^{1}\right) \cdot V\left(j u^{j-1}\right)\right)_{0} \\
& =(j-1)\left(V\left(\frac{\partial f}{\partial z_{0}}+j u^{j-1}\right) \cdot\left(\mathbb{C} \times \Gamma_{f, z_{0}}^{1}\right) \cdot V(u)\right)_{\mathbf{0}} \\
& =(j-1)\left(\Gamma_{f, z_{0}}^{1} \cdot V\left(\frac{\partial f}{\partial z_{0}}\right)\right)_{0} .
\end{aligned}
$$

iv) By [Lê1] or [Mas3], if $\Gamma_{h, L}^{1}$ is a curve, then

$$
\begin{aligned}
\left(\Gamma_{h, L}^{1} \cdot V(h)\right)_{0} & =\left(\Gamma_{h, L}^{1} \cdot V\left(L \frac{\partial h}{\partial L}\right)\right)_{0} \\
& =\left(\Gamma_{h, L}^{1} \cdot V(L)\right)_{0}+\left(\Gamma_{h, L}^{1} \cdot V\left(\frac{\partial h}{\partial L}\right)\right)_{0} .
\end{aligned}
$$

Therefore, iv) is a result of ii) and iii).

v) This follows at once by combining the prepolarity of $V\left(z_{0}\right)$ with Lemma 3.1 of [Mas2], which says that, for all large $j, \Sigma\left(f+z_{0}^{j}\right)=\Sigma f \cap V\left(z_{0}\right)$ near the origin.

Theorem 3.4. If $V\left(z_{0}\right)$ is a prepolar slice of $f$ at $\mathbf{0}$ then, for all large $j$,

$$
b_{n}\left(f+z_{0}^{j}\right)=b_{n}(f)-b_{n-1}(f)+j\left(b_{n-1}\left(f_{\left.\right|_{V\left(z_{0}\right)}}\right)-\left(\Gamma_{f, z_{0}}^{1} \cdot V\left(z_{0}\right)\right)_{0}\right) \text {. }
$$

In particular, $b_{n-1}\left(f_{\left.\right|_{V\left(z_{0}\right)}}\right) \geqslant\left(\Gamma_{f, z_{0}}^{1} \cdot V\left(z_{0}\right)\right)_{0}$.

Proof. After applying 2.2 to $f+u^{j}$ and the slice $V\left(z_{0}-u\right)$, we have

$$
b_{n+1}\left(f+u^{j}\right)-b_{n}\left(f+u^{j}\right)+b_{n}\left(f+z_{0}^{j}\right)=\left(\Gamma_{f+u^{j}, z_{0}-u}^{1} \cdot V\left(f+u^{j}\right)\right)_{0}
$$

which, by 3.3 , equals $j\left(\Gamma_{f, z_{0}}^{1} \cdot V\left(\frac{\partial f}{\partial z_{0}}\right)\right)_{0}$.

Now, as the Milnor fibre of $f+u^{j}$ has the homotopy-type of the one-point union of $j-1$ copies of the suspension of the Milnor fibre of $f$ [Mas3], [Ok], 
$[\mathbf{S a k}],[$ Se-Th$]$, we obtain

$$
(j-1) b_{n}(f)-(j-1) b_{n-1}(f)+b_{n}\left(f+z_{0}^{j}\right)=j\left(\Gamma_{f, z_{0}}^{1} \cdot V\left(\frac{\partial f}{\partial z_{0}}\right)\right)_{\mathbf{0}} .
$$

Using 2.2 on $f$ itself and rearranging, we get

$$
\begin{aligned}
b_{n}\left(f+z_{0}^{\jmath}\right) & =b_{n}(f)-b_{n-1}(f) \\
& +j\left[\left(\Gamma_{f, z_{0}}^{1} \cdot V\left(\frac{\partial f}{\partial z_{0}}\right)\right)_{0}-\left(\left(\Gamma_{f, z_{0}}^{1} \cdot V(f)\right)_{0}-b_{n-1}\left(f_{\left.\right|_{V\left(z_{0}\right)}}\right)\right)\right] .
\end{aligned}
$$

Finally, using once again (as in 3.3.iv) that

$$
\left(\Gamma_{f, z_{0}}^{1} \cdot V(f)\right)_{\mathbf{0}}=\left(\Gamma_{f, z_{0}}^{1} \cdot V\left(z_{0}\right)\right)_{\mathbf{0}}+\left(\Gamma_{f, z_{0}}^{1} \cdot V\left(\frac{\partial f}{\partial z_{0}}\right)\right)_{0},
$$

we obtain the desired result.

\section{Why Look at Prepolar Deformations?}

If $h$ is a complex analytic function, the Betti numbers of the Milnor fibre of $h$ at the origin are invariants of the ambient topological type of $V(h)$ at the origin and, hence, these Betti numbers are important data in the study of the topology of complex analytic singularities. Despite their importance, there is no known general method for calculating the Betti numbers of the Milnor fibre for a non-isolated hypersurface singularity. However, Theorem 3.4 provides some useful information: every prepolar deformation, $f$, of a fixed $f_{0}$ yields a lower bound on the top Betti number of the Milnor fibre of $f_{0}$.

In fact, dividing the equation of 3.4 by $j$ and taking limits, one obtains the interesting asymptotic formula

$$
b_{n-1}\left(f_{0}\right)=\left(\Gamma_{f, z_{0}}^{1} \cdot V\left(z_{0}\right)\right)_{\mathbf{o}}+\lim _{\jmath \rightarrow \infty} \frac{b_{n}\left(f+z_{0}^{j}\right)}{j} .
$$

One might hope that, by considering a prepolar deformation, $f$, for which $\left(\Gamma_{f, z_{0}}^{1} \cdot V\left(z_{0}\right)\right)_{0}$ obtains its maximal value, one would actually obtain the top Betti number of the Milnor fibre of $f_{0}$. This seems unlikely however; certain singularities seem to be "rigid" with respect to prepolar deformations - in the weak sense that any prepolar deformation, $f$, has no polar curve at the origin.

Nonetheless, the lower bounds provided by prepolar deformations give new data which helps describe the Milnor fibre of a completely general affine 
hypersurface singularity; this data does not appear to follow from our Morse inequalities between the Betti numbers of the Milnor fibre and the Lê numbers of the hypersurface, as described in [Mas1], [Mas2], and [Mas3].

As part of these Morse inequalities, we show that the 0 -th Lê number of $f_{0}, \lambda_{f_{0}}^{0}(\mathbf{0})$, provides an upper-bound on the top Betti number of the Milnor fibre of $f_{0}$. Also, we show in [Mas3] that if $f$ is a prepolar deformation of $f_{0}$, then

$$
\lambda_{f_{0}}^{0}(\mathbf{0})=\lambda_{f}^{1}(\mathbf{0})+\left(\Gamma_{f, z_{0}}^{1} \cdot V\left(z_{0}\right)\right)_{0},
$$

where $\lambda_{f}^{1}(\mathbf{0})$ is a Lê number of $f$ itself. Hence, for a fixed $f_{0}$, a prepolar deformation, $f$, with maximal $\left(\Gamma_{f, z_{0}}^{1} \cdot V\left(z_{0}\right)\right)_{0}$ will have minimal $\lambda_{f}^{1}(\mathbf{0})$. We prefer to call such a deformation a minimal prepolar deformation, instead of a maximal one.

It follows that, given a prepolar deformation, $f$, of $f_{0}$, the top Betti number of the Milnor fibre of $f_{0}$ is bounded by:

$$
\left(\Gamma_{f, z_{0}}^{1} \cdot V\left(z_{0}\right)\right)_{0} \leqslant b_{n-1}\left(f_{0}\right) \leqslant \lambda_{f}^{1}(\mathbf{0})+\left(\Gamma_{f, z_{0}}^{1} \cdot V\left(z_{0}\right)\right)_{0}
$$

and a minimal prepolar deformation will not only provide the maximal possible lower bound on the top Betti number of the Milnor fibre, it will also provide the smallest difference between our general upper and lower bounds.

One might hope that it is always possible to find a prepolar deformation, $f$, for which $\lambda_{f}^{1}(\mathbf{0})=0$, for then we would have $b_{n-1}\left(f_{0}\right)=\left(\Gamma_{f, z_{0}}^{1} \cdot V\left(z_{0}\right)\right)_{0}$; unfortunately, Proposition 1.28 of [Mas1] implies that it is usually impossible to find such a deformation.

One final comment: the inequality $b_{n-1}\left(f_{\left.\right|_{V\left(z_{0}\right)}}\right) \geqslant\left(\Gamma_{f, z_{0}}^{1} \cdot V\left(z_{0}\right)\right)_{0}$ appearing in Theorem 3.4 also follows from our Proposition 3.1 in [Mas1], where we describe a cancellation of handles in the Milnor fibre of $f$. However, this handle cancellation argument requires far more technical machinery than this paper does, and Proposition 3.1 of [Mas1] still does not yield the generalized Lê-Iomdine formula of Theorem 3.4.

\section{References}

[Fu] W. Fulton, Intersection Theory, Ergebnisse der Math., Springer-Verlag, 1984.

[H-L] H. Hamm and Lê D.T., Un Théorème de Zariski du type de Lefschetz, Ann. Sci. L'Ecole Norm. Sup., 6 (1973), 317-366.

[Io] I.N. Iomdin, Variétés complexes avec singularités de dimension un Sibirsk. Mat. Z., 15 (1974), 1061-1082. 
[Lê1] Lê D. T., Calcul du Nombre de Cycles Evanouissants d'une Hypersurface Complexe, Ann. Inst. Fourier, Grenoble, 23 (1973), 261-270.

[Lê2] Ensembles analytiques complexes avec lieu singulier de dimension un (d'après I.N. Jomdin), Sémin-aire sur les Singularités (Paris, 1976-1977) Publ. Math. Univ. Paris VII, (1980), 87-95.

[L-T] Lê D. T. and B. Teissier, Variétés polaires locales et classes de Chern des variétiés singulières, Annals of Math., 114 (1981), 457-491.

[Mas1] D. Massey, Lê Cycles and Hypersurface Singularities, monograph, 1994.

[Mas2] _ _ The Lê Varieties, I, Invent. Math., 99 (1990), 357-376.

[Mas3] _ The Lê Varieties, II, Invent. Math., 104 (1991), 113-148.

[M-S] D. Massey and D. Siersma, Deformations of Polar Methods, Ann. Inst. Four., 42 (1992), 737-778.

[Ok] M. Oka, On the homotopy type of hypersurfaces defined by weighted homogeneous polynomials, Topology, 12 (1973), 19-32.

[Sak] K. Sakamoto, The Seifert matrices of Milnor fiberings defined by holomorphic functions, J. Math. Soc. Japan, 26(4) (1974), 714-721.

[Se-Th] M. Sebastiani and R. Thom, Un résultat sur la monodromie, Invent. Math., 13 (1971), 90-96.

[Si-Tr] Y. T. Siu and G. Trautmann, Gap-Sheaves and Extension of Coherent Analytic Subsheaves, S.L.N. 172, Springer-Verlag, 1971.

[Te1] B. Teissier, Variétés polaires I: Invariants polaires des singularités d'hypersurfaces, Invent. Math., 40(3) (1977), 267-292.

[Te2] - Variétés polaires II: Multiplicités polaires, sections planes, et conditions de Whitney, in Algebraic Geometry, Proc., La Rabida 1981, Springer Lect. Notes, 961 (1982), 314-491.

Received February 15, 1994. The author was partially supported by National Science Foundation grant \#DMS-9003498.

NORTHEASTERN UNIVERSITY

Boston, MA 02115

E-mail address:dmassey@neu.edu 

Andreas Seeger, Endpoint inequalities for Bochner-Riesz multipliers in the

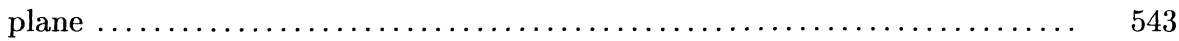

Ted Stanford, Braid commutators and Vassiliev invariants ............ 269

Xiangsheng $\mathbf{X u}$, On the Cauchy problem for a singular parabolic equation 277

Xingwang Xu, On the existence of extremal metrics .............. 555

Rugang Ye, Constant mean curvature foliation: singularity structure and

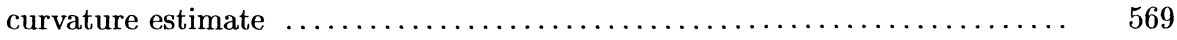




\section{PACIFIC JOURNAL OF MATHEMATICS}

Volume $174 \quad$ No. $2 \quad$ June 1996

Quantum affine algebras and affine Hecke algebras

VyJAYANTHI CHARI and ANDREW PRESSLEY

On the zero sets of bounded holomorphic functions in the bidisc

PHILIPPE CHARPENTIER and JOAQUIM ORTEGA-CERDÀ

Bloch constants in one and several variables

IAN GRAHAM and DROR VAROLIN

Characters of the centralizer algebras of mixed tensor representations of

$G L(r, \mathbb{C})$ and the quantum group $u_{q}(g l(r, \mathbb{C}))$

TOM HALVERSON

Derivations of $C^{*}$-algebras and almost Hermitian representations on

$\Pi_{k}$-spaces

EDWARD Kissin, ALEKSEI I. LOGINOV and Victor S. SHULman

Twisted Alexander polynomial and Reidemeister torsion

TERUAKI KITANO

Explicit solutions for the corona problem with Lipschitz data in the polydisc

STEVEN KRANTZ and SONG-YING Li

Prepolar deformations and a new Lê-Iomdine formula

DAVID MASSEY

$K K$-groups of twisted crossed products by groups acting on trees

Kevin Paul McClanahan

Coherent states, holomorphic extensions, and highest weight representations

KARL-HERMANN NEEB

Endpoint inequalities for Bochner-Riesz multipliers in the plane

ANDREAS SEEGER

On the existence of extremal metrics

XINGWANG XU

Constant mean curvature foliation: singularity structure and curvature estimate 569 RUGANG YE 